\title{
Tungíase: doença negligenciada causando patologia grave em uma favela de Fortaleza, Ceará
}

\author{
Tungiasis: a neglected disease causing severe morbidity in a shantytown in \\ Fortaleza, State of Ceará
}

\author{
Liana Ariza $^{1}$, Martin Seidenschwang ${ }^{2}$, John Buckendahl ${ }^{2}$, Marcia Gomide ${ }^{1}$, \\ Hermann Feldmeier ${ }^{2}$ e Jörg Heukelbach ${ }^{1,3}$
}

\begin{abstract}
RESUMO
A tungíase, ectoparasitose causada pela pulga Tunga penetrans, é endêmica em comunidades brasileiras de baixo poder aquisitivo. Neste estudo foram identificados babitantes de uma favela urbana em Fortaleza com carga parasitária elevada. Número de lesões, localização, estadiamento e patologias associadas foram registrados. Os 142 indivíduos identificados apresentaram condições de moradia extremamente precárias. Contou-se no total 3.445 lesões localizadas nos pés (mediana $=17$ lesões; máximo $=98$ lesões). Quase sem exceção, os indivíduos apresentaram deformações ungueais e edema e mais de $70 \%$ dor e fissuras. Perda de unha foi observada em $46 \%$ dos casos e deformação de dígitos em 25\%; $42 \%$ apresentaram abscessos e $59 \%$ queixaram-se de dificuldade de andar. Nossos dados mostram que a tungíase em comunidade urbana de baixa renda típica no nordeste brasileiro está associada a patologia grave. A doença precisa ser reconhecida como problema de saúde pública na região estudada e em outras áreas endêmicas semelbantes.
\end{abstract}

Palavras-chaves: Tungíase. Tunga penetrans. Ectoparasitose. Morbidade.

\section{ABSTRACT}

The parasitic skin disease tungiasis, caused by the jigger flea Tunga penetrans, is endemic in low-income communities in Brazil. In this study, inbabitants of a shantytown in Fortaleza, northeastern Brazil, who had an elevated parasite load, were identified. The number of lesions, localization, staging and associated diseases were recorded. The 142 individuals identified were living in extremely precarious housing conditions. A total of 3,445 lesions located on the feet were counted (median $=17$ lesions; maximum $=98$ lesions). Almost without exception, the individuals had nail deformation and edema, and more than $70 \%$ presented with pain and fissures. There was nail loss in 46\%; deformation of the digits in 25\%; abscesses in $42 \%$; and complaints of walking difficulty in $59 \%$. Our data show that tungiasis in this low-income urban community typical of northeastern Brazil was associated with severe morbidity. Tungiasis needs to be recognized as a public health problem in this study area and other similar endemic areas.

Key-words: Tungiasis. Tunga penetrans. Ectoparasite infestation. Morbidity.

A tungíase éuma doença ectoparasitária causada pela penetração da fêmea de Tunga penetrans (Linnaeus, 1758) na epiderme do seu hospedeiro. É uma pulga que hipertrofia subsequientemente até alcançar o tamanho de cerca de um centímetro ${ }^{15} 1926$.

Em contraste com as ectoparasitoses escabiose e pediculose, a tungíase é autolimitada com duração de quatro a seis semanas ${ }^{4}$. Nas áreas endêmicas, porém, a re-infestação constante é a regra, e os indivíduos afetados podem apresentar algumas dezenas de parasitos em diferentes estágios de desenvolvimento ${ }^{271024}$. Complicações graves e sequielas são comuns nessas áreas com baixos indicadores de desenvolvimento humano, onde as condições de higiene são precárias e a remoção da pulga não é realizada em condições de assepsia $^{719}$. Nessas circunstâncias, a superinfecção com bactérias patogênicas épresente sem exceção ${ }^{8}$, e a lesão causada pela penetração da pulga pode servir como porta de entrada para Clostridium tetani, agente causador do tétano ${ }^{132734} 35$. Seqüelas de infestação grave documentadas incluem dificuldade de andar, deformação e perda de unhas de dedo do pé, como também deformação e auto-amputação de dígitos, além de sepse e óbito ${ }^{31924282930}$.

Nos últimos anos, o número de publicações com o objetivo de aumentar a percepção da doença entre os gestores, profissionais de saúde e o mundo científico está aumentando, principalmente de países da América Latina e Caribe, como Argentina, Brasil e Haiti ${ }^{2} 111922243132$. Entretanto, relatos clínicos sistemáticos de

\footnotetext{
1. Departamento de Saúde Comunitária, Faculdade de Medicina, Universidade Federal do Ceará, Fortaleza, CE. 2. Instituto de Microbiologia Médica e Higiene, Faculdade de Medicina Charité, Berlim, Alemanha. 3. School of Public Health, Tropical Medicine and Rehabilitation Sciences, James Cook University, Townsville, Australia. Apoio financeiro: Intercâmbio Acadêmico PROBRAL nº152/02 (CAPES/DAAD) e CAPES (Bolsa de doutorado)

Endereço para correspondência: Prof. Jorg Heukelbach. Departamento de Saúde Comunitária/FM/UFC. R. Prof. Costa Mendes 1608/5andar, $60430-140$ Fortaleza, CE, Brasil. Tel: 5585 3366-8045. Fax: 5585 3366-8050.

e-mail: heukelbach@web.de

Recebido para publicação em 29/9/2006

Aceito em 17/1/2007
} 
pacientes com doença grave ainda são escassos, reforçando 0 fato de que a tungíase é uma doença negligenciada.

Aqui apresentamos as características clínico-epidemiológicas de indivíduos gravemente infestados com T. penetrans de uma área endêmica urbana típica brasileira. Os resultados mostram que a tungíase - de forma alarmante - está associada com patologia clínica.

\section{MATERIAL E MÉTODOS}

Área de estudo. 0 estudo foi realizado na área Serviluz/ Vicente Pinzón II, uma conglomeração de favelas urbanas típicas de Fortaleza, Ceará. Estas comunidades localizam-se nas dunas próximas à beira mar. Os migrantes pobres do interior do Estado começaram a habitar o local no início dos anos 50 do século XX. Atualmente, a área tem população total de aproximadamente 20.000 pessoas vivendo em condições sócio-econômicas precárias. Muitas casas são feitas de material reciclado e sem piso cimentado. Doenças parasitárias de pele como escabiose, larva migrans, pediculose e tungíase ocorrem freqüentemente nessa populaçã̃o $0^{21223}$.

População e desenho de estudo. A população examinada foi definida como parte de um estudo intervencional sobre a tungíase. Indivíduos com tungíase foram identificados com a ajuda de líderes comunitários e agentes de saúde. Foram incluídas somente pessoas com cinco ou mais lesões nos pés causadas por T. penetrans, baseando-se que mais de $90 \%$ das lesões encontram-se nos pés ${ }^{20}$. No total, 142 indivíduos de ambos os sexos foram recrutados das favelas Luxou, Morro do Sandra's, Morro da Vitória e Novo Rumo. Os participantes do estudo foram examinados clinicamente pela presença de tungíase em junho 2005. Esse mês encontra-se no período da seca, quando a doença ocorre mais frequentemente na área ${ }^{22}$. A localização e estágio das lesões foram documentados e os pacientes foram interrogados a respeito de sintomas utilizando uma ficha estruturada pré-testada. Foi também documentada a existência de patologia clínica aguda e crônica associada.

Exame clínico. A tungíase foi diagnosticada clinicamente de acordo com os critérios de Eisele e cols $s^{4}$ a) presença de uma pulga na fase de penetração ou de um pequeno ponto vermelhopardo com diâmetro de um a dois milímetros; b) presença de uma zona circular branca com diâmetro de três a dez milímetros com um ponto preto central; c) presença de uma casca preta (pulga morta com tecido necrosado). Os itens a) e b) foram definidos como lesões vitais e o item c) como lesão avital.

Após exame clínico, os sinais e sintomas de patologias associadas à tungíase foram observados e registrados, incluindo edema, eritema, dor, prurido, descamação, fissura e pústula. Complicações típicas de tungíase grave encontradas nos diferentes estágios de desenvolvimento da doença também foram registradas (deformação ou perda de unha, deformação de dedo, dificuldade de andar) ${ }^{47}$.

Armazenamento e análise dos dados. Os dados foram inseridos em uma base de dados usando o programa Epi Info (versão 6.04d; Centers for Disease Control and Prevention, Atlanta, USA) e checados por erros de digitação. Para comparar as medianas das lesões entre os grupos, os testes de Mann-Whitney e de Kruskal-Wallis foram utilizados.

Considerações éticas. Este estudo foi aprovado pelo Comitê de Ética em Pesquisa da Universidade Federal do Ceará (Protocolo COMEPE no. 21/2003). Participaram do estudo apenas os indivíduos ou responsáveis legais que assinaram o termo de consentimento. Após exame clínico, todos os participantes da pesquisa foram tratados contra a tungíase com um repelente natural à base de óleo de côco por um período de, pelo menos, quatro semanas. Esse repelente se mostrou efetivo em reduzir a penetração de pulgas em indivíduos com infestação grave? .

\section{RESULTADOS}

Características sócio-demográficas individuais e familiares. No total foram incluídos 142 indivíduos com pelo menos cinco lesões causadas por fêmeas da T. penetrans. Destes, 69 (48,6\%) eram de sexo masculino e 73 (51,4\%) feminino. A mediana da idade foi de nove anos, sendo os limites oito meses e 66 anos.

0s 142 participantes do estudo procederam de 73 famílias residentes na área. A maioria das casas possuía energia elétrica (97,3\%) e abastecimento de água por rede geral (83,6\%). Contudo, não havia rede de esgoto e de águas servidas. Quarenta e sete $(64,3 \%)$ das casas eram feitas de madeira ou material aproveitado, e $37(50,7 \%)$ tinham piso de areia. Na maioria $(56 / 76,7 \%)$ das famílias, não tinha nenhum membro com 0 primeiro grau completo. A mediana da renda familiar mensal foi de $R \$ 119,00$ (intervalo interquartil $\mathrm{R} \$ 60,00$ a $R \$ 200,00$ ).

Localização das lesões e número de lesões (carga parasitária). 0 número de lesões (total, vitais, avitais e manipuladas por indivíduo) está indicado na Tabela 1. Ao todo foram contadas 3.445 lesões nos 142 indivíduos (mediana $=17$ ); $11,3 \%$ (16/142) apresentaram mais de 50 lesões. 0 máximo de 98 lesões foi encontrado em uma menina de sete anos. Não houve diferença significativa do número mediano de lesões entre o sexo masculino e feminino ( 16 vs $17 ; p=0,4)$. 0 número mediano do total das lesões entre os grupos etários não mostrou diferença estatisticamente significativa $(\mathrm{p}=0,19)$. Entretanto, a mediana das lesões manipuladas foi maior nos indivíduos acima de 20 anos do que nos abaixo dessa idade ( 7 vs $4 ; \mathrm{p}=0,002$ ). Cinqüenta e uma $(35,9 \%)$ lesões apresentavam-se de forma agrupada (em cluster, Figuras 1 e 2). A distribuição topográfica das lesões está sumarizada na Tabela 2.

Tabela 1 - Número total de lesões e distribuição de acordo com estágios, em uma favela urbana de Fortaleza, $\mathrm{CE}$.

\begin{tabular}{lccc}
\hline & \multicolumn{3}{c}{ Número de lesões } \\
\cline { 2 - 4 } Estágios & Mediana & IIQ** & Máximo \\
\hline Total & 17 & $12-30$ & 98 \\
Vitais* & 6 & $4-11$ & 74 \\
Avitais* & 6 & $3-11$ & 49 \\
Manipuladas & 4,5 & $3-8,5$ & 50 \\
\hline
\end{tabular}

* de acordo com Eisele e cols ${ }^{4}$

*** Intervalo interquartil 


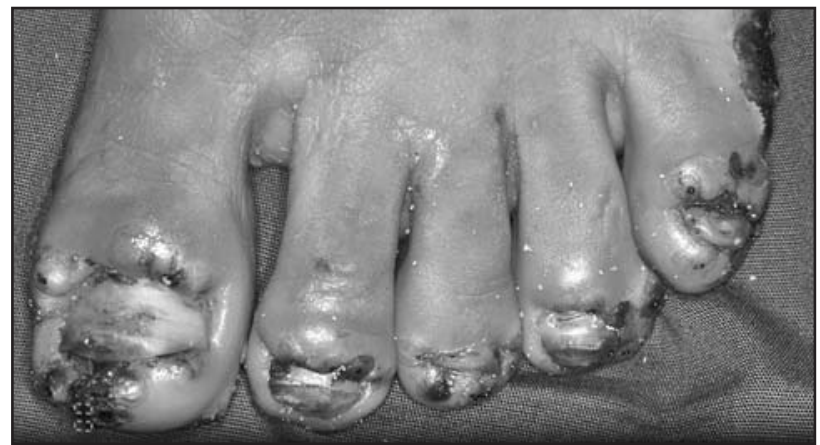

Figura 1 - Pé esquerdo de uma de menina de sete anos. Os dedos apresentam lesões em todos os estágios de desenvolvimento. Por exemplo, o bálux apresenta cluster de lesões vitais no canto proximal da unha e cluster de lesões em estágios vitais e avitais na área distal do dedo. Ovos podem ser identificados aderidos à pele e em todas as unhas (e.g., seis ovos no bordo lateral da unba do $4^{\circ}$ pododáctilo). Inflamação está presente em todos os dedos; todas as unbas e o $5^{\circ}$ pododáctilo apresentam deformação.

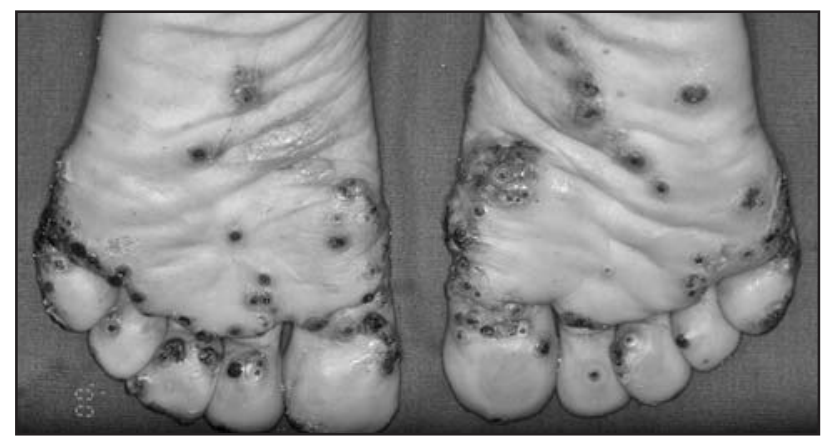

Figura 2 - Região plantar dos pés de uma menina de sete anos. Lesões em todos os estágios de desenvolvimento estão presentes, como também sinais de lesões antigas tipo punched out (e.g., na área proximal ao $5^{\circ}$ dedo pododáctilo do pé esquerdo). Vários agrupamentos de lesões (clusters) são visíveis em ambos os pés.

Tabela 2 - Distribuição do número de lesões nos pés dos indivíduos examinados ( $\mathrm{n}=142$ ), em uma favela urbana de Fortaleza, $\mathrm{CE}$.

\begin{tabular}{|c|c|c|c|c|c|c|}
\hline \multirow[b]{2}{*}{ Localização } & \multicolumn{2}{|c|}{ Indivíduos } & \multicolumn{4}{|c|}{ Número de lesões } \\
\hline & $\mathrm{n}^{\underline{0}}$ & $\%$ & $\mathrm{n}^{\underline{0}}$ & $\%$ & Mediana & IIQ* \\
\hline Dedos & 141 & 99,3 & 2416 & 70,1 & 14 & $9-20$ \\
\hline peri-ungueal & 137 & 96,5 & 1864 & 54,1 & 11 & $7-17$ \\
\hline não peri-ungueal & 103 & 72,5 & 552 & 16,0 & 2 & $0-2$ \\
\hline Planta & 88 & 62,0 & 501 & 14,5 & 1 & $0-5$ \\
\hline Calcanhar & 57 & 40,1 & 341 & 9,9 & 0 & $0-5$ \\
\hline Canto Lateral & 33 & 23,2 & 187 & 5,4 & 0 & $0-0$ \\
\hline
\end{tabular}

* Intervalo interquartil

Patologia e sintomatologia associadas. As patologias e sintomatologias associadas com tungíase - diferenciadas em aguda e crônica - estão indicadas na Tabela 3. Praticamente, todos os participantes mostraram deformação de, pelo menos, uma unha e edema (Figura 1). A maioria se queixou de prurido e dor. De forma relevante, fissuras associadas a lesões foram encontradas em mais do que $2 / 3$ dos indivíduos. Quase a metade perdeu pelo menos uma unha, e um quarto apresentou-se com deformação de dígitos, como seqüela crônica de infestação repetida e permanente (Figura 1). Mais da metade (59\%) tinha dificuldade de andar.
Tabela 3 - Sintomas e patologias agudas e crônicas associadas a tungíase identificadas na população do estudo $(n=142)$ em uma favela urbana de Fortaleza, $\mathrm{CE}$.

\begin{tabular}{lcc}
\hline & \multicolumn{2}{c}{ Indivíduos } \\
\cline { 2 - 3 } Sintomas e patologia & $\mathrm{n}^{-}$ & $\%$ \\
\hline Agudos & 133 & 93,7 \\
edema e eritema & 114 & 80,3 \\
prurido & 101 & 71,1 \\
dor espontânea & 100 & 70,9 \\
dor a pressão* & 98 & 69,0 \\
fissura & 83 & 58,9 \\
dificuldade de andar* & 59 & 41,6 \\
abscesso/supuração & 38 & 26,8 \\
úlceras & & \\
Crônicos & 141 & 99,3 \\
deformação de unha & 132 & 93,0 \\
hiperceratose & 131 & 92,3 \\
descamação & 129 & 90,8 \\
sinais de lesões antigas** & 122 & 85,9 \\
hipertrofia peri-ungueal & 65 & 45,8 \\
perda de unha & 35 & 24,6 \\
deformação de dígitos & 24 & 16,9 \\
pele brilhante & & \\
\hline
\end{tabular}

* dados disponíveis de 141 indivíduos. *** lesões residuais punched out conforme descritas em Eisele e cols ${ }^{4}$.

\section{DISCUSSÃO}

Os dados descritos mostram que a tungíase, ainda hoje, é causa de patologia grave em uma comunidade urbana com baixos indicadores de desenvolvimento humano, e que nessas condições essa ectoparasitose precisa ser considerada como um importante problema de saúde pública. Como a área de estudo se assemelha às tantas outras favelas urbanas do Nordeste brasileiro, é possível supor que problema de igual gravidade seja bastante comum nessa região do país.

A tungíase é um exemplo evidente de uma doença associada à pobreza ${ }^{18} 1924$. Muehlen e cols ${ }^{31}$ mostraram que as condições precárias de construção da habitação, a baixa escolaridade, a presença de animais e o baixo nível sócio-econômico são os fatores mais importantes associados à presença de tungíase grave. Desde os anos 70 do século XX, a prevalência da doença vem decrescendo em muitas áreas, mas continua sendo alarmente em inúmeras comunidades desfavorecidas do Brasil $^{2714151932}$. Recentemente, a Organização Mundial de Saúde considerou importante o impacto negativo causado por doenças ectoparasitárias na qualidade de vida ${ }^{5}$, sugerindo que no futuro essa ectoparasitose seja reconhecida de forma mais cuidadosa.

Neste estudo, as patologias associadas à tungíase são alarmantes. Incômodos como prurido e dor estavam presentes e mais da metade dos examinados tinha dificuldade em andar. Fissuras nos pés, que podem servir como porta de entrada para microorganismos patogênos, ocorreram em mais de $2 / 3$ dos indivíduos examinados. De fato, foi relatado que no Brasil e em outros países a tungíase está associada a um amplo espectro 
de patologias clínicas agudas e crônicas ${ }^{671236}$. A superinfecção bacteriana das lesões é uma constante e sua gravidade pode resultar em seqüelas como gangrena, auto-amputação de dígitos, sepse e tétano ${ }^{7} 1925273435$. Recentemente, em estudo realizado no país mais pobre das Américas, Haiti, houve relato de quatro óbitos em adultos extremamente infestados, provavelmente devido ao tétano e/ou a sepse ${ }^{24}$.

Esses relatos são preocupantes na medida em que a tungíase é uma doença autolimitada, que se agrava com as precárias condições de vida gerando seqüelas e desfechos potencialmente evitáveis.

Até o momento, o tratamento padrão consiste em remover a pulga com uma agulha estéril e aplicação de um antibiótico tópico ${ }^{15}{ }^{19}$. A extração da pulga penetrada requer habilidade manual, instrumental adequado e condições de higiene satisfatórias - os quais raramente são disponíveis nas áreas endêmicas - do contrário pode causar mais danos do que benefícios`. Além desses aspectos, a extração das pulgas não é um procedimento tão viável nas comunidades altamente afetadas, nas quais a taxa de ataque pode ser superior a dez pulgas por indivíduo, diariamente ${ }^{22}$.

Nos últimos anos, estudos clínicos controlados com uso tópico de ivermectina, tiabendazol e metrifonate mostraram pequeno efeito $^{16}$. 0 uso de ivermectina oral também não se mostrou eficaz quando comparado ao placebo $^{17}$. Recentemente, estudos mostraram que um repelente à base de óleo de côco, também recomendado contra picadas de insetos e carrapatos, é altamente eficaz tanto na regressão da patologia clínica de indivíduos gravemente infestados, como na prevenção de re-infestaçãa $0^{933}$. 0 uso desse repelente talvez seja a medida mais adequada para reduzir a patologia associada à tungíase do que o tratamento após infestação ${ }^{1522}$.

Concluímos que independe dos esforços que têm sido realizados para o manejo clínico da tungíase, o controle sustentável requer o empenho dos gestores públicos de saúde na implementação de ações de controle e monitoramento no âmbito coletivo, aliado às melhorias da educação, do trabalho e da infraestrutura urbana nas comunidades mais carentes.

\section{AGRADECIMENTOS}

À comunidade Serviluz/Vicente Pizón II, e aos líderes comunitários Liduina, Francisco "Quico", e Euzanira. À secretária Valéria Assunção, e aos assistentes de campo Marilene da Silva Paulo, Maria de Fátima Cavalcante, Mavel, Daniele Mendonça, Maria José Nascimento, Francisco José Nascimento, Aurilene da Silva Paulo e José Maria.

\section{REFERÊNCIAS}

1. Cardoso AEC. Tunguíase. Anais Brasileiros de Dermatologia 65: 29S-33S, 1990 .

2. Carvalho RW, Almeida AB, Barbosa-Silva SC, Amorim M, Ribeiro PC, Serra-Freire NM. The patterns of tungiasis in Araruama township, state of Rio de Janeiro, Brazil. Memórias do Instituto Oswaldo Cruz 98: 31-36, 2003

3. Chadee DD. Tungiasis among five communities in south-western Trinidad, West Indies. Annals of Tropical Medicine and Parasitology 92: 107-113, 1998.
4. Eisele M, Heukelbach J, van Marck E, Mehlhorn H, Meckes 0, Franck S, Feldmeier H. Investigations on the biology, epidemiology, pathology and control of Tunga penetrans in Brazil: I. Natural history of tungiasis in man. Parasitology Research 90: 87-99, 2003.

5. Engels D, Savioli L. Reconsidering the underestimated burden caused by neglected tropical diseases. Trends in Parasitology 22: 364-366, 2006.

6. Falkenstein. Über das Verhalten der Haut in den Tropen, ihre Pflege und Krankheiten. Virchows Archiv Pathologische Anatomie 71: 421-440, 1877.

7. Feldmeier H, Eisele M, Saboia-Moura RC, Heukelbach J. Severe tungiasis in underprivileged communities: case series from Brazil. Emerging Infectious Diseases 9: 949-955, 2003

8. Feldmeier H, Heukelbach J, Eisele M, Sousa AQ, Barbosa LM, Carvalho CB. Bacterial superinfection in human tungiasis. Tropical Medicine and International Health 7: 559-564, 2002.

9. Feldmeier H, Kehr JD, Heukelbach J. A plant-based repellent protects against Tunga penetrans infestation and sand flea disease. Acta Tropica 99: 126-136, 2006

10. Feldmeier H, Kehr JD, Poggensee G, Heukelbach J. High exposure to Tunga penetrans (Linnaeus, 1758) correlates with intensity of infestation. Memórias do Instituto Oswaldo Cruz 101: 65-69, 2006.

11. González A, Villalobos C, Ranalletta MA, Coscarón MC. Aspectos adaptativos y biológicos de Tunga penetrans (Linné, 1758). Epidemiologia en comunidades aborígenes del norte argentino. Archivos Argentinos de Dermatologia 54: 119$123,2004$.

12. Gordon RM. The jigger flea. Lancet 2: 47-49, 1941

13. Greco JB, Sacramento E, Tavares-Neto J. Chronic Ulcers and Myasis as Ports of Entry for Clostridium tetani. Brazilian Journal of Infectious Diseases 5: 319-323, 2001.

14. Heukelbach J. Tungiasis. Revista do Instituto de Medicina Tropical de São Paulo 47: 307-313, 2005.

15. Heukelbach J. Revision on tungiasis: treatment options and prevention. Expert Review of Anti-Infective Therapy 4: 151-157, 2006.

16. Heukelbach J, Eisele M, Jackson A, Feldmeier H. Topical treatment of tungiasis: a randomized, controlled trial. Annals of Tropical Medicine and Parasitology 97: 743-749, 2003.

17. Heukelbach J, Franck S, Feldmeier H. Therapy of tungiasis: a double-blinded randomized controlled trial with oral ivermectin. Memórias do Instituto Oswaldo Cruz 99: 873-876, 2004.

18. Heukelbach J, Mencke N, Feldmeier H. Editorial: Cutaneous larva migrans and tungiasis: the challenge to control zoonotic ectoparasitoses associated with poverty. Tropical Medicine and International Health 7: 907-910, 2002.

19. Heukelbach J, Oliveira FA, Hesse G, Feldmeier H. Tungiasis: a neglected health problem of poor communities. Tropical Medicine and International Health 6 : 267-272, 2001.

20. Heukelbach J, Wilcke T, Eisele M, Feldmeier H. Ectopic localization of tungiasis. American Journal of Tropical Medicine and Hygiene 67: 214-216, 2002.

21. Heukelbach J, Wilcke T, Feldmeier H. Cutaneous larva migrans (creeping eruption) in an urban slum in Brazil. International Journal of Dermatology 43: $511-515,2004$

22. Heukelbach J, Wilcke T, Harms G, Feldmeier H. Seasonal variation of tungiasis in an endemic community. American Journal of Tropical Medicine and Hygiene 72: 145-149, 2005.

23. Heukelbach J, Wilcke T, Winter B, Feldmeier H. Epidemiology and morbidity of scabies and pediculosis capitis in resource-poor communities in Brazil. British Journal of Dermatology 153: 150-156, 2005.

24. Joseph JK, Bazile J, Mutter J, Shin S, Ruddle A, Ivers L, Lyon E, Farmer P. Tungiasis in rural Haiti: a community-based response. Transactions of the Royal Society of Tropical Medicine and Hygiene 100: 970-974, 2006.

25. Joyeux C, Sicé A. Précis de médecine coloniale. Masson, Paris, 1937.

26. Linardi PM. Família Tungidae. In: Linardi PM, Guimaraes LR (eds) Sifonápteros do Brasil. Museu de Zoologia da Universidade de São Paulo, São Paulo, p.48-53; 2000.

27. Litvoc J, Leite RM, Katz G. Aspectos epidemiológicos do tétano no Estado de São Paulo (Brasil). Revista do Instituto de Medicina Tropical de São Paulo 33: 477484, 1991. 
28. Mashek H, Licznerski B, Pincus S. Tungiasis in New York. International Journal of Dermatology 36: 276-278, 1997.

29. Matias RS. Epidemia de tungíase no Rio Grande do Sul. Revista da Sociedade Brasileira de Medicina Tropical 22: 137-142, 1989.

30. Melo CR, Melo IS. Linfedema elefantóide verrucoso associado à infestação maciça por Tunga penetrans. Anais Brasileiros de Dermatologia 64: 35-37, 1989.

31. Muehlen M, Feldmeier H, Wilcke T, Winter B, Heukelbach J. Identifying risk factors for tungiasis and heavy infestation in a resource-poor community in Northeast Brazil. Transactions of the Royal Society of Tropical Medicine and Hygiene 100: 371-380, 2006.

32. Muehlen M, Heukelbach J, Wilcke T, Winter B, Mehlhorn H, Feldmeier H. Investigations on the biology, epidemiology, pathology and control of Tunga penetrans in Brazil II. Prevalence, parasite load and topographic distribution of lesions in the population of a traditional fishing village. Parasitology Research 90: 449-455, 2003.

33. Schwalfenberg S, Witt LH, Kehr JD, Feldmeier H, Heukelbach J. Prevention of tungiasis using a biological repellent: a small case series. Annals of Tropical Medicine and Parasitology 98: 89-94, 2004.

34. Soria MF, Capri JJ. Tetanos y "piques". La Prensa Medica Argentina 40: 4-11, 1953.

35. Tonge BL. Tetanus from chigger flea sores. Journal of Tropical Pediatrics 35: 94 , 1989

36. Valença ZO, Cardoso AEC, Cardoso AS. Tunguiase generalizada: relato de dois casos tratados com thiabendazol. Dermatologia Ibero-Latino-Americana 3: 375-378, 1972. 